\title{
Parameters for Automatic Modulation Recognition of HF Signals
}

\author{
J. Giesbrecht* ${ }^{\dagger}$, R. Clarke ${ }^{\dagger}$, D. Abbott* \\ *University of Adelaide \\ Adelaide, SA 5000, Australia \\ (Email: james@eleceng.adelaide.edu.au, dabbott@eleceng.adelaide.edu.au) \\ ${ }^{\dagger}$ Ebor Computing \\ 147 Henley Beach Road, Mile End, SA 5031, Australia \\ (Tel: +61 882380300 Email: james.giesbrecht@ebor.com, russell.clarke@ebor.com)
}

Keywords: Coherence, Entropic Distance, Entropy, SNR, Modulation Recognition.

\begin{abstract}
Two fundamental processes of modulation recognition are parameter extraction and feature selection. Parameter extraction determines unique characteristics of the signal such as frequency, phase, and amplitude. Parameters may also include statistical measures such as standard deviation, and $\mathrm{n}^{\text {th }}$-order moments and cumulants. This paper provides a brief review of two parameters, coherence and entropic distance, and re-introduces a statistical measure of signal-to-noise ratio (SNR). It also discusses the usefulness of these parameters in automatic modulation recognition of HF signals.
\end{abstract}

\section{Introduction}

A tendency of modern communication systems (very often at frequencies above $800 \mathrm{MHz}$ ) is to achieve high bandwidth communications by moving to higher and higher frequencies. This push often comes with a perception that high-frequency (HF) communications is obsolete. High-frequency communications is alive, however, and indeed undergoing resurgence despite the advances of modern communications. Spectrum mangement organisations monitor the HF spectrum to control and enforce licensing of HF users, while defence agencies are interested in the HF spectrum, with respect to surveillance and back-up communications. These activities usually require systems capable of determining the location of a source of transmission, separating valid signals from interference and noise, and identification of signal type (or modulation recognition).

Modulation recognition generally comprises three major steps: parameter extraction, feature selection, and classification. Sometimes parameter extraction and feature selection are combined and called feature extraction.

Parameter extraction attempts to isolate unique characteristics of the signal so that the signal can be classified. Fundamental characteristics are frequency, phase, and amplitude, but statistical measures (e.g. standard deviation, $\mathrm{n}^{\text {th }}$-order moments, $\mathrm{n}^{\text {th }}$-order cumulants) are not uncommon. Whatever the parameters, they are chosen to form an $N$-dimensional vector.

Feature selection then transforms the $N$-dimensional parameter space into an $M$-dimensional feature space, where $M \leq N$. With an optimal choice of parameters, the $M$ dimensional feature space consists of $M$ orthogonal basis vectors. This may not be possible for a sub-optimal parameter list, but the more orthogonal the basis vectors the easier a classifier can separate signal types.

The role of classification is to identify the modulation type based on the selected features. Associations are normally made through statistical methods, decision theoretic methods, artificial neural networks (ANNs), or pattern recognition algorithms.

Our focus is on the combined feature extraction step. So, what are useful features for automatically recognising a given HF signal?

\section{Parameters for Modulation Recognition}

Amongst the literature there are many parameters under investigation for the purposes of modulation recognition $[1,2,4,5,13,14,15,17,18,19,20]$. They include characteristics such as power-spectral density (PSD), signal-noise ratio (SNR), bandwidth, instantaneous frequency and amplitude, and statistical measures (including high-order moments and cumulants).

Research described in the literature suffers problems related to assumptions about the transmission medium and methods of validation. In most cases, an assumption of Gaussian noise is made, which is rarely valid in practice $[11,16]$. And few, if any of the papers, apply modulation recognition algorithms to real signals. In our context, real implies signals propagating by multiple modes with co-channel interference and nonGaussian noise. In fact, it is well known, that HF noise is impulsive [11,16]. 
Our previous work $[6,7,8,9,10]$ discusses coherence and entropic distance in detail, however we briefly review these parameters here. We also re-introduce a parameter, first proposed by Aisbett [1], which estimates the true power of a signal contaminated by noise. For the sake of a name, we call this parameter the Aisbett Hash Function. With a slight alegrabic modification, the function can also be used to estimate SNR.

\subsection{Coherence}

The coherence function [4] (sometimes called the magnitude squared coherence) is a ratio of power spectral densities and provides a measure of the similarity of two signals at specific frequencies. It is analogous to correlation in the frequency domain. Given two signals, $X$ and $Y$, the coherence is defined as

$$
\gamma^{2}(f)=\left|\frac{P_{x y}(f)}{\sqrt{P_{x x}(f) P_{y y}(f)}}\right|^{2}
$$

where $P_{x x}(f)$ is the auto-power spectral density of signal $X$, $P_{y y}(f)$ is the auto-power spectral density of signal $Y$, and $P_{x y}(f)$ is the cross-power spectral density of signals $X$ and $Y$.

At each frequency, the coherence function varies between 0 and 1 and indicates the similarity between $X$ and $Y$. If the value of the function at a particular frequency is close to unity, it indicates that $X$ and $Y$ are similar at that frequency. On the other hand, if the value of the function is near zero it implies that the two signals are dissimilar at the particular frequency.

In a practical sense, the computation of coherence is difficult. Carter [4] suggests the use of a overlapped windowing method, such as Welch's [22] averaged periodogram method, to estimate the coherence. Welch's method breaks a digitised signal (hereafter referred to as a signal segment) into overlapping sections. Each overlapping section is detrended and smoothed by an $n$-point window (e.g. Hanning, Hamming) before an $n$-point Fast Fourier Transform (FFT) is applied.

Coherence is sensitive to misalignment of time signals, number of overlapping sections, percentage of overlap, and phase. The coherence function is therefore not useful for FSK or PSK type signals because it is affected by the message carried by the signals $[8,9,10]$. At best, the coherence function is useful only for signals with highly correlated messages.

\subsection{Entropic Distance}

Shannon [21] defined entropy as a measure of the uncertainty of the occurrence of an event or the information that event imparts when it occurs. Benedetto, Caglioti, and Loreto [3] base their definition of entropy on Shannon's formulation but present a different method of computing it. They create a measure of the similarity or dissimilarity of two information sources, $\mathbf{A}$ and $\mathbf{B}$, as representative of the true entropy. The method compresses a long sequence $A$ from $\mathbf{A}$ and subtracts this compressed length from the length of the compressed sequence $A+b$, where $A+b$ is the concatenation of $A$ and a small sequence $b$ from $\mathbf{B}$. This is the entropy of $\mathbf{A}$ (designated by $\Delta_{A b}$ ). In a similar manner they compute the entropy of $\mathbf{B}$ as $\Delta_{B a}$ and the self-entropies $\Delta_{A a}$ and $\Delta_{B b}$. They then define the relative entropy between $\mathbf{A}$ and $\mathbf{B}$ as

$$
S_{A B}=\frac{\Delta_{A b}-\Delta_{B b}}{|b|},
$$

where $|b|$ is the length of $b$, and the relative entropy between $\mathbf{B}$ and $\mathbf{A}$ as

$$
S_{B A}=\frac{\Delta_{B a}-\Delta_{A a}}{|a|},
$$

where $|a|$ is the length of $a$. The total entropy, or entropic distance, between the two information sources is then by definition

$$
S_{T} \equiv \frac{\Delta_{A b}-\Delta_{B b}}{\Delta_{B b}}+\frac{\Delta_{B a}-\Delta_{A a}}{\Delta_{A a}}
$$

Entropic distance is analogous to a measure of the difficulty that an English-speaking person has in learning Chinese or the difficulty that a Chinese-speaking person has in learning English.

Our previous work $[10,12]$ has demonstrated that entropic distance can be used to separate real HF groundwave signals, specifically the FSK modes of Mil-Std-188-110A (i.e. FSK Wideband, FSK Alternate Wideband, FSK Narrowband), and Stanag 4285 (an 8-PSK signal).

\subsection{Aisbett Hash Function}

The Aisbett Hash Function is the product of the means of two signals less their covariance. The name of the function is derived from the way that Aisbett [1] specified the function; $\#(X, Y)$ was used in place of the more common $f(X, Y)$ representation of a function.

Given two digitised signals in the time domain, $X$ and $Y$, the Aisbett Hash Function is

$$
\begin{aligned}
& \#(X, Y)= \frac{2}{N^{2}} \sum_{j=1}^{N} X(j T) \sum_{k=1}^{N} Y(k T) \\
&-\frac{1}{N} \sum_{m=1}^{N} X(m T) Y(m T) \\
& \#(X, Y)=2 E\{X\} E\{Y\}-E\{X Y\},
\end{aligned}
$$


where $T$ is the sample period, $N$ is the number of samples in each sequence, and $E\{\}$ is the expectation operator.

From (5b), an estimate of signal power is simple to compute. For a given received passband signal it can be shown that its envelope, denoted by $A(t)$, is

$$
A(t)=B(t) \cos \phi(t)+n(t),
$$

where $B(t)$ is the envelope of the modulating signal, $\phi(t)$ is the phase of the modulating signal, $n(t)$ is zero-mean narrowband Gaussian noise, and $t$ is time. To estimate signal power one computes

$$
\#\left(A^{2}, A^{2}\right)=2 E\left\{A^{2}\right\} E\left\{A^{2}\right\}-E\left\{A^{2} \cdot A^{2}\right\} .
$$

Aisbett shows that (7) is an unbiased estimator of signal power for constant-envelope signals. With a constant envelope signal

$$
E\left\{A^{2}(t)\right\}=B^{2}(t)+2 \sigma^{2}
$$

where $\sigma^{2}$ is the Gaussian noise power. Equation (8) represents the power of the baseband signal plus the power of the noise. Then since $\#\left(A^{2}, A^{2}\right)$ is proportional to $B^{2}(t)$, the estimate of $\mathrm{SNR}_{\mathrm{e}}$ can be written as

$$
\mathrm{SNR}_{\mathrm{e}}=K \frac{\#\left(A^{2}, A^{2}\right)}{E\left\{A^{2}(t)\right\}-\#\left(A^{2}, A^{2}\right)},
$$

where $K$ is a normalization constant. For the present discussion $K=1 / 2$.

\section{Performance of the Aisbett Hash Function}

We generate five signals with added Gaussian noise namely, 2-FSK, 4-FSK, 2-PSK, 4-PSK, and Stanag 4285 (8-PSK). Though our goal is to apply parameters to real HF signals with non-Gaussian noise and co-channel interference, for the current discussion Gaussian noise is sufficient. Next we apply (9) to the synthetic signals at various SNRs.

Figure 1 illustrates the true signal plus noise power for each signal versus SNR. For an SNR greater than $5 \mathrm{~dB}$ the noise has little impact on the true signal plus noise power thus showing that the true signal power for each signal is $0.5 \mathrm{~W}$. Figure 2 shows that $\#\left(A^{2}, A^{2}\right)$ closely estimates the true signal power for the constant envelope signals at a true SNR greater than $-3 \mathrm{~dB}$. However, $\#\left(A^{2}, A^{2}\right)$ underestimates the power of the Stanag 4285 signal by a factor of approximately four. This is expected because ( $5 \mathrm{~b}$ ) provides an estimate of the magnitude of the envelope of a signal; for Stanag 4285 the envelope varies and so (5b) tends toward zero.

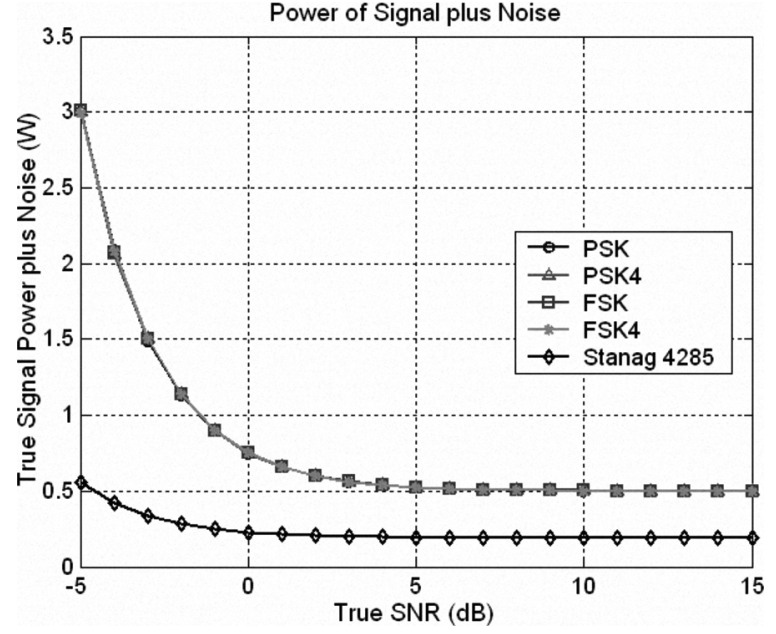

Figure 1: True signal power plus noise power for each of the synthetic signals (i.e. 2-PSK, 4-PSK, 2-FSK, 4-FSK, and Stanag 4285) with added Gaussian noise.

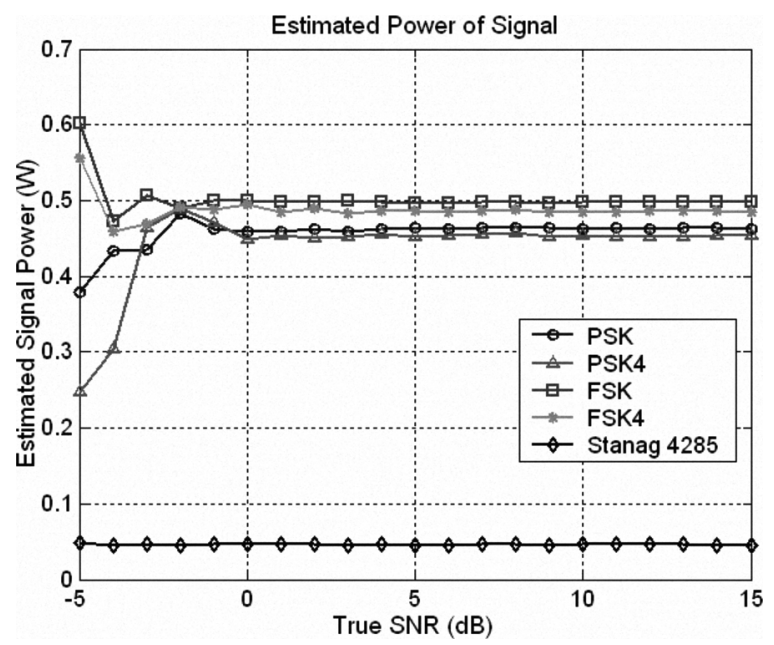

Figure 2: Estimate of signal power computed with $\#\left(A^{2}, A^{2}\right)$. Only for low SNR does \# $\left(A^{2}, A^{2}\right)$ not provide a good estimate of signal power.

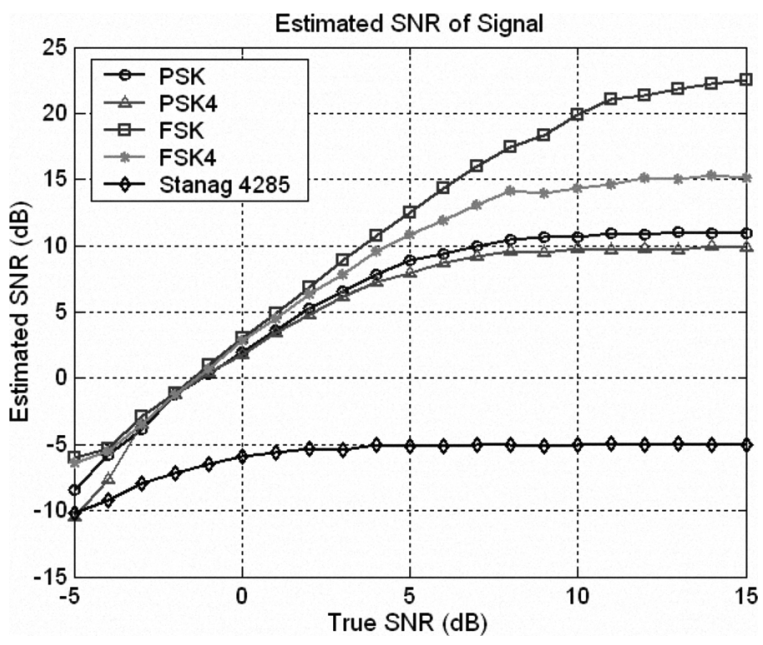

Figure 3: Estimated SNR for synthetic signals with zeromean Gaussian noise. Note the linear portion of the curves and their eventual independence of the true SNR. 
Equation (9) does appear to provide useful information about each signal. For certain ranges, Figure 3 shows that estimated SNR follows an approximately linear (in a logarithmic sense) relationship with the true SNR. For 2-FSK this region is about $15 \mathrm{~dB}$ along the abcissa. For Stanag 4285 the region is only about $5 \mathrm{~dB}$. The linear range for all the other signals is about $10 \mathrm{~dB}$. Thus within a limited range and with suitable scaling, Equation (9) can provide a reasonable estimate of SNR.

What is more interesting is that direct application of (9) appears to separate the signals when the true SNR is high. The tendency towards saturation as the true SNR increases is a result of the noise power decreasing exponentially with a constant envelope not equal to one. If the magnitude of the envelope, $B(t)$, of the modulation signal is unity then a true linear relationship exists between the estimated SNR and the true SNR. Consider (8) and that

$$
E\left\{A^{4}(t)\right\}=B^{4}(t)+8 \sigma^{2} B^{2}(t)+8 \sigma^{4} .
$$

Combining (7), (8), (9), and (10) results in

$$
\mathrm{SNR}_{\mathrm{e}}=K B^{2}(t) \frac{\frac{B^{2}(t)}{\sigma^{2}}}{\frac{B^{2}(t)}{\sigma^{2}}\left(1-B^{2}(t)\right)+2}
$$

and

$$
\mathrm{SNR}_{\mathrm{e}}=K B^{2}(t) \frac{\mathrm{SNR}_{\mathrm{T}}}{\left(1-B^{2}(t)\right) \mathrm{SNR}_{\mathrm{T}}+2}
$$

which says that for a constant envelope signal, with $B^{2}(t) \neq 1$, the estimated SNR, $\mathrm{SNR}_{e}$, is non-linear with respect to the true SNR, SNR . The asymptotes in Figure 3 are thus related to the magnitudes of their respective signal envelopes. Of course, if $B^{2}(t)=1$, then $(11 \mathrm{~b})$ reduces to the linear equation

$$
\mathrm{SNR}_{\mathrm{e}}=\frac{K}{2} \mathrm{SNR}_{\mathrm{T}} .
$$

Thus the separation of curves, in Figure 3, is a due to differences in the magnitudes of the envelopes of the modulation signals. To maximise the linear range over which (9) is useful, the magnitude of $B(t)$ in the received signal must be maintained as close to unity as possible. Automatic gain control (AGC) could be applied to the baseband signal prior to application of (9) but, in practice, it will be difficult to ensure that $B(t)$ is near unity at low SNR. Morever, the separation of curves is related to the amplitude of a baseband signal and therefore (9) cannot be used to unambigously separate constant envelope signals.

In fact, if the simulation is repeated with all signals normalized to span the entire range of -1 to +1 (thus ensuring that $\left.B^{2}(t)=1\right)$ the curves in Figure 3 fall upon each other in Figure 4 . The Stanag 4285 signal does not have a constant envelope and so its estimate of SNR does not follow the trend of the other signals.

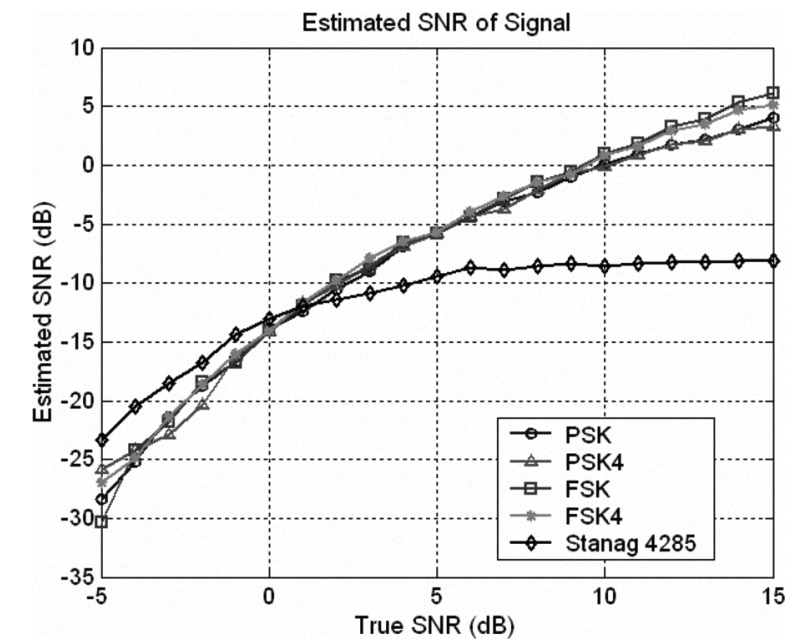

Figure 4: Estimated SNR of various synthetic signals with zero-mean Gaussian noise. In this case all curves align, save that of Stanag 4285. This is a result of ensuring that each signal spans the entire range of -1 to +1 .

\section{Further Work}

Literature searches and experience with real HF signals leads to the following suggested set of parameters to recognize the target signals: centre frequency, bandwidth, SNR [1], envelope, symbol frequencies, cross-Margenau-Hill disbribution [17], kurtosis [2], signal constellation, modulation level, auto-regressive-covariance [17], entropic distance $[9,10,12]$, and power spectral density.

The Cross-Margenau-Hill Distribution (CMHD), which is related to the cross-correlation function, may separate $m$-ary PSK signals while an auto-regressive covariance function may separate $m$-ary FSK signals. Kurtosis provides promise in separating BPSK, QPSK, QAM, and multi-tone signals. Entropic distance also appears able to separate real $\mathrm{HF}$ signals $[9,10,12]$. Other parameters that aid modulation recognition are centre frequency, bandwidth, SNR, signal envelope, symbol frequencies, constellation, and power spectral densities.

Our immediate future work will be to apply the Aisbett Hash Function to real HF signals, where real refers to signals propagating by multiple ionospheric modes with co-channel interference and non-Gaussian noise. Signals of interest include $m$-ary FSK, $m$-ary PSK, multi-tone PSK, Stanag 4285, and Mil-Std-188-110A signals.

\section{Summary}

This paper reviewed coherence and entropic distance, and summarised their suitability as paramaters for automatic modulation recognition of HF signals. It also introduced a method to estimate signal-to-noise ratio (SNR). Coherence is 
unsatisfactory for identifying either FSK or PSK signals. Coherence is useful only for signals with highly correlated messages. On the other hand, entropic distance is useful for separating the FSK modes of Mil-Std-188-110A and Stanag 4285 (8-PSK). Aisbett's [1] hash function shows promise for estimating the signal-to-noise (SNR) of a signal, but not for direct identification of an unknown signal.

\section{Acknowledgements}

The University of Adelaide and Ebor Computing provide funding for this work.

\section{References}

[1] J. Aisbett. Automatic Modulation Recognition Using Time Domain Parameters. Technical Report ERL-0367TR, Electronic Research Laboratory DSTO, Department of Defence, Commonwealth Government of Australia, November 1986.

[2] W. Akmouche. Detection of multicarrier modulations using 4th-order cumulants. In Proc. of IEEE MILCOM 1999 Military Communications Conference, volume 1, pages 432-436, Atlantic City, NJ, USA, November 1999.

[3] D. Benedetto, E. Caglioti, and V. Loreto. Language trees and zipping. Physical Review Letters, 88(4), 2002.

[4] G. C. Carter. Coherence and Time Delay Estimation: An Applied Tutorial for Research, Development, Test, and Evaluation Engineers. IEEE Press, Piscataway, NJ, 1993.

[5] E. Choi and C. Lee. Feature extraction based on the Bhattacharyya distance. Pattern Recognition, 36:17031709, 2003.

[6] J. E. Giesbrecht, R. Clarke, and D. Abbott. Improved techniques for monitoring the HF spectrum. In Proc. of SPIE International Symposium on Microelectronics, $M E M S$, and Nanotechnology, volume 5274, pages 112122, Perth, Australia, 2004.

[7] J. E. Giesbrecht, R. Clarke, and D. Abbott. Monitoring the HF spectrum in the presence of noise. In Proc. of SPIE Second International Symposium on Fluctuations and Noise, volume 5473, pages 76-84, Maspalomas, Gran Canaria, Spain, 2004.

[8] J. E. Giesbrecht, R. Clarke, and D. Abbott. Coherence as a feature of real HF signals. In Proc. of SPIE Third International Symposium on Fluctuations and Noise, volume 5847, pages 188-198, Austin TX, USA, 2005.

[9] J. E. Giesbrecht, R. Clarke, and D. Abbott. Modulation recognition for HF signals. In Proc. of SPIE International Symposium on Microelectronics, MEMS, and Nanotechnology, volume 5649, pages 501-512, Sydney, Australia, 2005.

[10] J. E. Giesbrecht, R. Clarke, and D. Abbott. Modulation recognition for real HF signals. In Proc. of SPIE International Symposium on Microelectronics, MEMS, and Nanotechnology, volume 6035, pages 458-469, Brisbane, Australia, 2005.

[11] J. E. Giesbrecht, R. Clarke, and D. Abbott. An empirical study of the probability density function of HF noise.
Fluctuations and Noise Letters, 6(2):L117-L125, March 2006. World Scientific Publishing Company.

[12] J. Giesbrecht, R. Clarke, and D. Abbott. Entropic distance for automatic modulation recognition of $\mathrm{HF}$ signals. IEEE Transactions on Wireless Communications, publication pending.

[13] A. O. Hero III and H. Hadinejad-Mahram. Digital modulation classification using power moment matrices. In Proc. of the IEEE 1998 International Conference on Acoustics, Speech, and Signal Processing, volume 6, pages 3285-3288, Seattle, 1998.

[14] R. D. Hippenstiel and P. M. De Oliveira. Time-varying Spectral Estimation using the Instantaneous Power Spectrum (IPS). In IEEE Transactions on Signal Processing, volume 38, pages 1752-1759. Institute of Electrical and Electronic Engineers, October 1990.

[15] S.-Z. Hsue and S. S. Soliman. Automatic modulation classification using zero crossing. IEE Proceedings, 137(6):459-464, December 1990.

[16]E. E. Johnson, R. I. Desourdis Jr., G. D. Earle, S. C. Cook, and J. C. Ostergaard. Advanced High-Frequency Radio Communications. Artech House Inc., Norwood MA, USA, 1997.

[17]H. Ketterer, F. Jondral, and A. H. Costa. Classification of modulation models using time-frequency methods. In Proceedings of the IEEE 1999 International Conference on Acoustics, Speech, and Signal Processing, volume 5, pages 2471-2474, Phoenix, Arizona, 1999.

[18] R. A. Mitchell and J. J. Westerkamp. Robust Statistical Feature Based Aircraft Identification. IEEE Transactions on Aerospace and Electronic Systems, 35(3):1077-1093, 1999.

[19] A. K. Nandi and E. E. Azzouz. Modulation recognition using artificial neural networks. Signal Processing, 56:165-175, 1997.

[20]A. K. Nandi and E. E. Azzouz. Algorithms for Automatic Modulation Recognition of Communication Signals. IEEE Transactions on Communications, 46(4):431-435, April 1998.

[21]C. E. Shannon. A Mathematical Theory of Communication. Bell System Technical Journal, 27:379423, 623-656, July, October 1948.

[22]P. D. Welch. The use of the fast fourier transform for the estimation of power spectra: A method based on time averaging over short, modified periodograms. IEEE Transactions on Audio and Electroacoustics, AU-15:7073, June 1967 